\title{
TOMOGRAPHIC-QUALITY PHASE CALIBRATION VIA PHASE CENTER DOUBLE LOCALIZATION
}

\author{
Stefano Tebaldini, Mauro Mariotti d'Alessandro, Francesco Banda, Claudio Prati \\ Politecnico di Milano - DEIB
}

\begin{abstract}
In this paper we propose a strategy for phase calibrating repeat-pass Synthetic Aperture Radar (SAR) data for tomographic applications. The problem is tackled by observing that multi-baseline data provide equations enough to jointly estimate aircraft and target positions, up to a rototranslation of the coordinate system used for representing the imaged scene. Such a rototranslation is shown to correspond to the forward operator null space, which can be accounted for in order to project the results in the desired coordinate frame. Volumetric scatterers are treated by exploiting the Phase Linking algorithm, which allows to represent them as equivalent phase centers. Results are shown from synthetic data and real SAR data acquired over snow and ice.
\end{abstract}

\section{INTRODUCTION}

Synthetic Aperture Radar (SAR) Tomography (TomSAR) has been receiving increasing attention in the last years by different research groups, [1], [2], [3]. TomSAR provides access to the three-dimensional (3D) structure of the imaged scene, yielding most useful information both in forestry and urban applications. TomSAR processing is in principle a straightforward extension of conventional SAR focusing from the $2 \mathrm{D}$ to the $3 \mathrm{D}$ space. The signal gathered along a single flight line is focused through integration along a 1D synthetic aperture, resulting in 2D (range, azimuth) resolution capabilities. By collecting multiple flight lines the signal can be integrated over a 2D synthetic aperture, resulting in 3D resolution capabilities. The fundamental requirement for this simple approach to work is that the knowledge about the flown flight lines is accurate enough to predict variations of the sensor-to-target distances to within an accuracy much better than the system wavelength, which is typically on the order of few to few tenths of centimeters. For this reason current airborne SAR systems employ sophisticated navigation equipment, including GPS and inertial sensors, to record platform motion. The performance of such systems is often enough to correctly predict the distance variation along a single flight line, thus ensuring correct signal focusing in the 2D space. Yet, it does not provide the required sub-wavelength accuracy concerning the location of one flight line with respect to another. This translates into residual phase screens that appear in SAR interferograms as low-frequency phase modulations [4], [5]. Such phase screens may easily turn out to be critical for TomSAR imaging, possible effects ranging from side-lobes to complete defocusing [5]. For this reason, both SAR Interferometry (InSAR) and TomSAR require a preprocessing phase calibration step. This problem was tackled in many works in the last years, that differ from each other based on how phase screens are estimated and parametrized. A possible approach is to phase calibrate the data by removing the interferometric phases associated with ground scattering, which are estimated based on detected stable point targets within the data-stacks [5], or by isolating ground-only contributions via a polarimetric decomposition techniques [6]. This approach allows a consistent reconstruction of volumetric scattering above the ground. Yet, terrain topography remains unknown unless dedicated InSAR procedures are employed [7]. A similar approach is found in [8], with the difference that the residual phase screens are obtained via entropy minimization. A different perspective is found in [4]. In that paper a procedure is proposed for the estimation of the alongtrack derivatives of motion errors in a single-baseline interferogram, which are afterwards integrated and used to correct the data.

In this paper we propose a novel approach to phase calibration of multi-baseline airborne SAR data by recasting the problem in terms of jointly estimating aircraft and target phase center positions, which we will hereinafter refer to as Phase Center Double Localization. This approach entails different advantages over existing phase calibration algorithms, such as: i) low computational burden; ii) no need for phase unwrapping nor parameter integration; iii) no need for point targets; iv) no need for detectable ground contributions; $v$ ) it works on single polarization data; vi) it yields a consistent reconstructions of volumetric scattering above the ground and terrain topography.

\section{MULTI-BASELINE PHASE CALIBRATION}

The phase calibration procedure proposed in this paper is based upon two key concepts:

- multi-baseline data provide equations enough to jointly estimate aircraft positions and target phase center height at each along track position (Double Localization), up to a roto-translation of the coordinate system;

- volumetric targets can be represented as equivalent phase centers by means of a suitable phase model.

\subsection{Double Localization}

Consider a planar (2D) scenario where $N$ sensors and $P$ targets are present, as depicted in figure 1, left panel. The double localization problem is cast in terms estimating the sensor and target positions in the $2 \mathrm{D}$ space based on the set of distances $R_{n}^{p}$ from each sensor to each target. It is immediate to see that the set of distances is invariant with respect to any distance-preserving coordinate transformation. Accordingly, the solution will be defined up to a roto-translation of the coordinate system. To fix coordinate translation we will assume one reference sensor whose position is known. This implies that the uncertainty about the targets is reduced to their elevation (i.e.: angular position) with respect to the reference sensor, since distances are known. To fix coordinate rotation we will then assume the angular position of one target with respect to the reference sensor is known. The question is now whether it is possible to solve for the remaining 

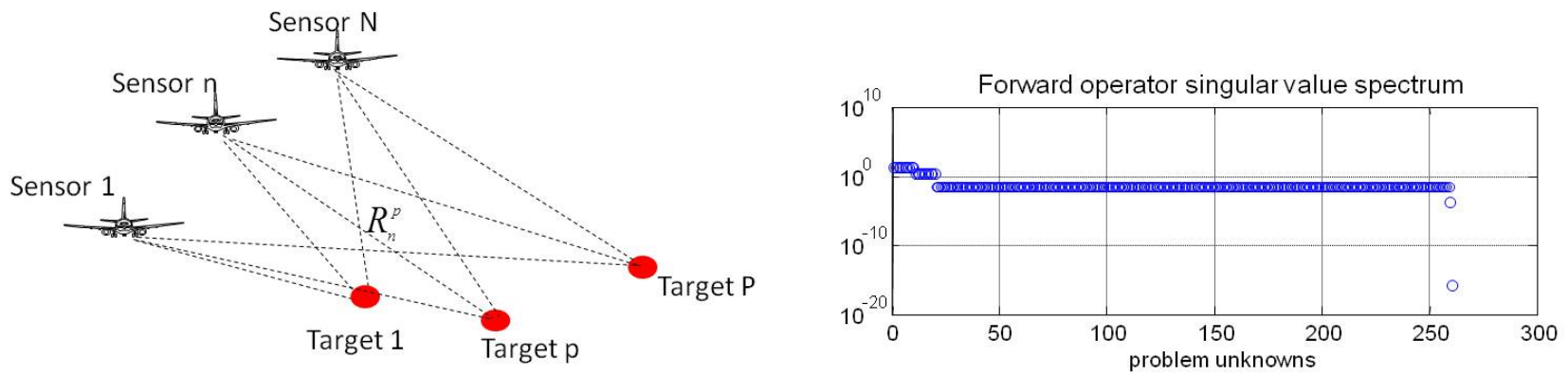

Fig. 1. Left panel: reference scenario for the double localization problem. Right panel: Singular value spectrum for the problem forward operator defined in eq. (1). The last singular value is on the order of $10^{-16}$, corresponding to the operator null space.
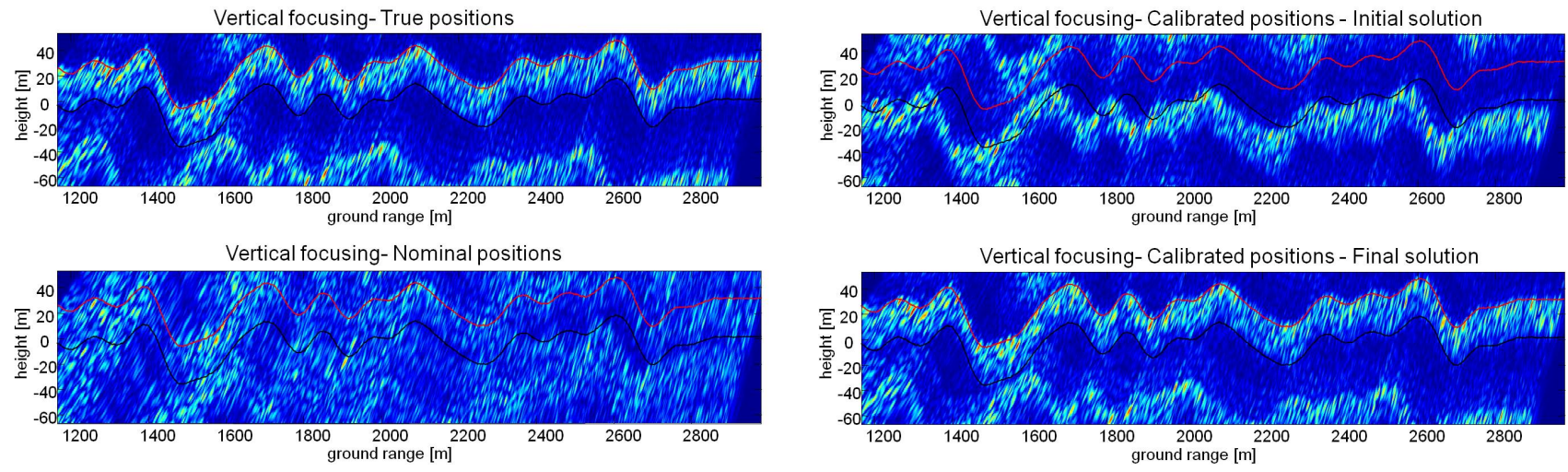

Fig. 2. Focused images along an azimuth cut (single-look intensity). Top left: true sensors positions. Bottom left: wrong sensor positions. Top right: First solution to which the algorithm converged. Bottom right: final solution after null space reinsertion. The black and red lines indicate terrain topography and volume top height, respectively.

unknowns in this reference frame. To answer this question we assume a nominal solution and consider the expression of the distance derivatives about this solution:

$$
d R_{n}^{p}=b_{n}^{p} d \theta^{p}-d Y_{n} \sin \theta^{p}+d Z_{n} \cos \theta^{p}
$$

where: $\theta^{p}$ is the nominal incidence angle at $p ; b_{n}^{p}$ is the nominal normal baseline for the $n-t h$ flight at position $p ; d \theta^{p}$ is the elevation error for the target at position $p ; d Y_{n}$ and $d Z_{n}$ are the baseline errors for the $n-t h$ flight. The unknowns in equation (1) are represented by target elevation error $d \theta^{p}$ and the baseline errors $d Y_{n}, d Z_{n}$. Equation (1) therefore corresponds to a linear system of $N_{e}=(N-1) P$ equations in $N_{x}=2(N-1)+P-1$ unknowns. The condition $N_{e} \geq N_{x}$ cannot be verified unless $N \geq 3, P \geq 3$. Accordingly, a necessary condition to solve this problem is that at least 3 sensors are available, and of course at least 3 targets as well. This condition can also be proved to be sufficient by analyzing the singular values of the problem forward operator defined in eq. (1). An example of this analysis is shown in the rightmost panel of figure 1 . In this case the problem was parametrized with $2(N-1)$ unknowns for sensor positions and $P$ unknowns for target elevation. As a result, the problem null space dimension turns out to be exactly 1 , corresponding to the fact that coordinate rotation was not fixed.

\subsection{Equivalent Phase Center}

The distance set from all sensors to all targets, can be derived with great accuracy from the multi-baseline data stack itself. To this aim, consider a set of $N$ SAR acquisitions focused on a common reference $2 \mathrm{D}$ grid, and define $d_{n}^{p}$ as the complex valued pixel in the $n-t h$ image of the data stack at position $p$ along a fixed along-track (azimuth) position, where $p$ may indicate either the slant or ground range coordinate, depending on the grid used for focusing. The interferometric phase with respect to a reference track (Master) can then be expressed as:

$$
\varphi_{n}^{p}=\angle d_{n}^{p} d_{r e f}^{p *}=\frac{4 \pi}{\lambda} d R_{n}^{p}
$$

where $\lambda$ is the carrier wavelength. The main factor hindering the employment of this relation to solve the double localization problem above is that it implies that the concept of target position is well defined. This is surely true when dealing with point or superficial targets, whereas it is not obvious to define target position for a generic volumetric target. Assuming equation (2) the phase of the $n m-t h$ interferogram should obey to [9]:

$$
\varphi_{n m}^{p}=\angle d_{n}^{p} d_{m}^{p *}=\frac{4 \pi}{\lambda}\left(d R_{n}^{p}-d R_{m}^{p}\right)=\varphi_{n}^{p}-\varphi_{m}^{p}
$$



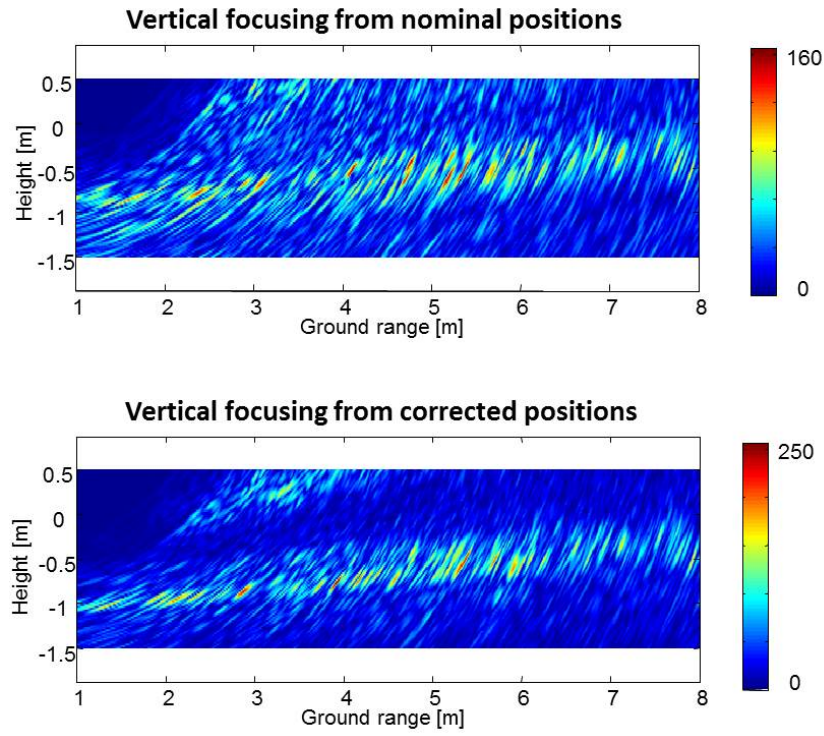

Fig. 3. Ground based SAR Tomography of the snow pack at Col de Porte, French Alps. Vertical focusing along a constant-azimuth cut. Top panel: focusing based on nominal antenna positions. Bottom panel: focusing based on corrected antenna positions. The color scale represents signal intensity.

that is, assuming target position is well defined entails that the interferometric phases enjoy the remarkable property expressed in equation (3), which allows to describe the phases of all available interferograms through just $N-1$ numbers [9]. This property is in general lost when dealing with volumetric targets, due to both the spatial structure and the temporal behavior of the target. In this case, however, retaining equation (3) can be interpreted as a way of approximating a volumetric target to an equivalent phase center whose position is well defined. The big advantage of this approach the $N-1$ phases $\varphi_{n}^{p}$ representing the target as a phase center can be estimated straightforwardly from all available interferograms based on the Phase Linking algorithm proposed in [9]. The meaningfulness of this approach will be instead discussed on an experimental basis in the next section.

\section{RESULTS FROM SYNTHETIC DATA}

A synthetic data-set was produced by simulating $N=10$ airborne SAR acquisition over a $30 \mathrm{~m}$ thick volume of random scatterers on an topographic terrain. The height of ambiguity is slightly higher than twice the volume height. The resulting interferometric coherences vary between 0.1 and 0.8 , depending on the normal baseline value. The data was focused along a constant azimuth cut by assuming the true sensor positions, see the top left panel of figure 2, and by simulating random positioning errors with a standard deviation of $30 \mathrm{~cm}$, see the bottom left panel of the same figure. Phase calibration was performed according to the following steps: $i$ ) ground-range signal focusing according based on nominal (i.e.: wrong) sensor positions; ii) interferogram multi-looking; iii) estimation of the $N-1$ phases via the Phase Linking algorithm; iv) solution of the Double Localization problem in $(1) ; v$ ) signal focusing in the ground-range, height plane based on the estimated sensor positions. The Double Localization problem was solved by iteratively alternating the esti-
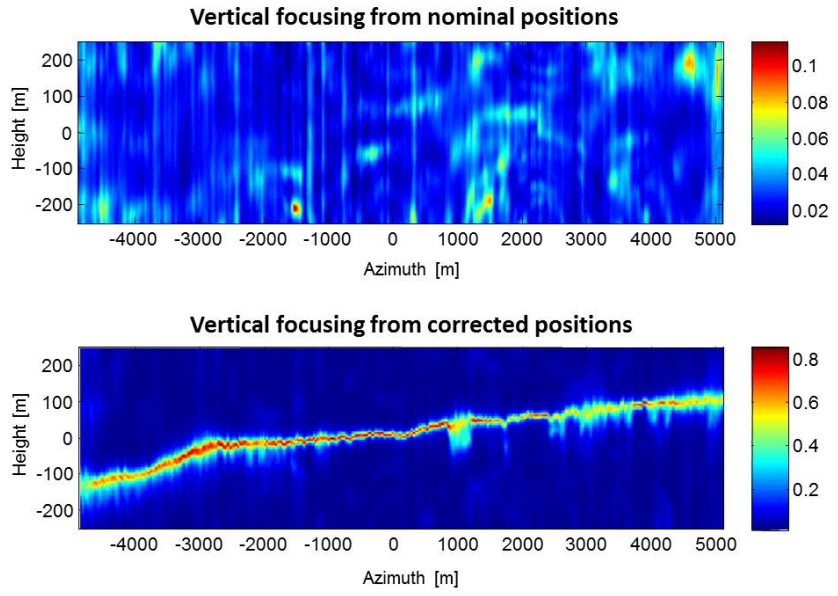

Fig. 4. Airborne SAR Tomography of the ice layer in the area of Kangerlussuaq, Greenland. Vertical focusing along a constant-range cut. Top panel: focusing based on nominal antenna positions. Bottom panel: focusing based on corrected antenna positions. The color scale represents signal intensity.

mation of sensor and target positions in the complex domain, without the need for phase unwrapping. The resulting focused image is shown in the top right panel of figure 2 . It is immediate to see that this image is affected by a global coordinate transformation with respect to the one obtained by assuming perfect knowledge of sensor positions. Yet, the target vertical structure is reconstructed nearly without error. This is perfectly consistent with the analysis of section 2.1, where it was shown that in absence of information about the target a one dimensional null space arises that correspond to a global coordinate rotation. This residual coordinate transformation can be compensated for by operating on the left singular vectors of the forward operator, which can be easily fixed in presence of any kind of external information about target position (like average terrain slope, for example). The obvious way to do this is to use the last left singular vector, which rigorously corresponds to the problem null space. Yet, the solution can be made more robust by considering also singular vectors associated with the lowest singular values, as discussed in [7]. This options should be considered with care as it strongly depends on the problem forward operator, that is on acquisition geometry. The final image after this correction is shown in the bottom right panel figure 2 , where it is easy to note that both the target vertical structure and terrain topography are nearly perfectly recovered. This result also validates the idea presented in section 2.2, according to which the Phase Linking algorithm can be used to approximate volumetric targets as equivalent phase centers.

\section{RESULTS FROM REAL DATA}

In this section we present results from real data-sets acquired over snow and ice. The first data-set here considered was acquired during a Ground Based (GB) SAR campaign carried out by the University of Rennes I in December 2010 at Col de Porte, in the French Alps, in collaboration with Meteo-France, to the aim of investigating the vertical structure of a $60 \mathrm{~cm}$ snow-pack. Signal transmission and reception are controlled by a Vector Network Analyzer (VNA) moved by a stepper motor along a $3 \mathrm{~m}$ rail. Ten acquisitions were gathered by varying the rail height, in order to provide $3 \mathrm{D}$ resolution capa- 
bilities. The system was operated in the frequency range between 8.2 GHz and $16.2 \mathrm{GHz}$ (X and $\mathrm{Ku}-\mathrm{Band}$ ), corresponding to a total transmitted bandwidth of $8 \mathrm{GHz}$. The resulting spatial resolution provided by this configuration is about $2 \mathrm{~cm}$ in range (across track), $2 \mathrm{~cm}$ azimuth (along track), and $8 \mathrm{~cm}$ in cross-range (orthogonal to the across- and along-track directions), which allows to image the snow-pack to a sufficiently fine resolution so as to reveal its vertical structure. The second data-sets was collected in the frame of the ESA campaign IceSAR in 2012, aimed of investigating ice motion and vertical structure in the area of Kangerlussuaq, Greenland. During this campaign, P-band SAR data were acquired by the Technical University of Denmark (DTU) with the airborne system POLARIS, which was originally developed as a nadir looking ice sounder but recently upgraded with a SAR capability. The data-set available for TomSAR is constituted by 10 passes acquired along two different directions, obtained by flying POLARIS along an oval-like racetrack. Results are shown in figures (3) and (4). Calibrated images are observed to exhibit high contrast and detail level in both cases, whereas uncalibrated images are clearly affected by defocusing phenomena. In the GB SAR case image degradation is limited to the presence of side-lobes, which arise due to offsets about the rail positions on the order of few millimeters, see the top panel of figure (3). In the airborne case the uncalibrated image is instead completely defocused, due to the fact that the relative position of one flight line with respect to another is not known to within an accuracy comparable with the system wavelength, see the top panel of figure (4).

\section{CONCLUSIONS}

Errors about antenna phase center positions that are not negligible compared to system wavelength have a critical impact on multibaseline SAR tomography, possible effects ranging from side-lobes to complete defocusing [5]. In this paper we have proposed a processing strategy to calibrate multi-baseline SAR data by recasting the problem in terms of jointly estimating aircraft and target phase center positions. The proposed approach has been shown to provide a consistent reconstructions of volumetric scattering above the ground and terrain topography. The algorithm does not require the presence of point-like or superficial targets, as it is able to operate on (temporally stable) volumetric targets. The fundamental condition for the applicability of this methodology is that each image is correlated with at least another one, so as to enable phase center estimation using the the Phase Linking algorithm. For a generic volumetric scenario this condition is fulfilled as long as volume height is lower than the system height of ambiguity, that is as long as system geometry has been designed correctly. Current researches are focused on the development of a multi-squint version of the Phase Linking algorithm, which also embeds models of the aircraft dynamics.

\section{ACKNOWLEDGMENTS}

The authors wish to heartily thank prof. Laurent Ferro-Famil (University of Rennes I) and Prof. Jorgen Dall (DTU) for the data presented in this paper, as well as for insights and valuable discussions.

\section{REFERENCES}

[1] A. Reigber and A. Moreira, "First demonstration of airborne SAR tomography using multibaseline 1-band data," IEEE Trans. on Geoscience and Remote Sensing, pp. 2142-2152, Sep. 2000.
[2] S. Tebaldini and F. Rocca, "Multibaseline polarimetric sar tomography of a boreal forest at P- and L-bands," Geoscience and Remote Sensing, IEEE Transactions on, vol. 50, no. 1, pp. 232 -246, jan. 2012.

[3] O. Frey and E. Meier, "Analyzing tomographic sar data of a forest with respect to frequency, polarization, and focusing technique," Geoscience and Remote Sensing, IEEE Transactions on, vol. 49, no. 10, pp. 3648 -3659, october 2011.

[4] A. Reigber, P. Prats, and J. Mallorqui, "Refined estimation of time-varying baseline errors in airborne sar interferometry," Geoscience and Remote Sensing Letters, IEEE, vol. 3, no. 1, pp. 145 - 149, jan. 2006.

[5] S. Tebaldini and A. Guarnieri, "On the role of phase stability in sar multibaseline applications," Geoscience and Remote Sensing, IEEE Transactions on, vol. 48, no. 7, pp. 2953 -2966, 2010.

[6] S. Tebaldini and F. Rocca, "On the impact of propagation disturbances on SAR tomography: Analysis and compensation," in Radar Conference, 2009 IEEE, 4-8 2009, pp. 1 -6.

[7] G. Gatti, S. Tebaldini, M. Mariotti d'Alessandro, and F. Rocca, "Algae: A fast algebraic estimation of interferogram phase offsets in space-varying geometries," Geoscience and Remote Sensing, IEEE Transactions on, vol. 49, no. 6, pp. 2343 -2353, june 2011.

[8] M. Pardini, K. Papathanassiou, V. Bianco, and A. Iodice, "Phase calibration of multibaseline sar data based on a minimum entropy criterion," in Geoscience and Remote Sensing Symposium (IGARSS), 2012 IEEE International, july 2012, pp. 5198 -5201.

[9] A. M. Guarnieri and S. Tebaldini, "On the exploitation of target statistics for SAR interferometry applications," Geoscience and Remote Sensing, IEEE Transactions on, vol. 46, no. 11, pp. 3436-3443, Nov. 2008. 